\title{
Sparse convolutional plane-wave compounding for ultrasound imaging
}

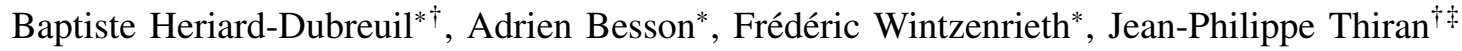 \\ and Claude Cohen-Bacrie* \\ *E-Scopics, Saint-Cannat, France \\ ${ }^{\dagger}$ Signal Processing Laboratory (LTS5), Ecole polytechnique fédérale de Lausanne (EPFL), Lausanne, Switzerland \\ $¥$ Department of Radiology, University Hospital Center (CHUV) and University of Lausanne (UNIL), Lausanne, Switzerland \\ Email: firstname.lastname@e-scopics.com or firstname.lastname@epfl.ch
}

\begin{abstract}
Ultrafast ultrasound imaging enables imaging at kilohertz frame rates, at the cost of a degraded image quality in terms of contrast and resolution. To reach an image quality comparable to focused imaging, the standard technique is coherent compounding, which requires a high number of transmissions and reduces the effective frame rate. In this paper, we introduce a COnvolutional Compounding Algorithm (COCA), a non-linear compounding method that aims at improving the quality of plane-wave imaging by virtually creating new angles. It enables to perform high-quality imaging with a significantly reduced number of emissions. Tests carried out on simulations, in vivo and in vitro experiments, using the Plane-wave Imaging Challenge in Medical UltraSound (PICMUS) dataset, show a significant improvement in terms of contrast and resolution compared to coherent compounding, increasing lateral resolution by 15 to $30 \%$ and contrast by $6.8 \mathrm{~dB}$ in average, reaching an overall quality almost comparable to a coherent compounding of 75 angles with only 7 angles.
\end{abstract}

Index Terms-Convolutional compounding; non-linear beamformer; plane wave imaging.

\section{INTRODUCTION}

Ultrafast Ultrasound imaging is a recently proposed technique that aims at increasing dramatically the frame rate, reaching the kilohertz range where the standard imaging method, focused imaging, was limited to $30-40 \mathrm{~Hz}$ ([1], [2]). This technique is based on the emission of unfocused plane waves that can insonify the whole medium and give access to one image per wave. Due to the lack of focusing, image quality is reduced, but can be restored using several plane waves with different angles, via coherent compounding, as shown in [3], [4]. However, the number of plane waves required to reach a decent image quality is high, reducing the effective frame rate.

The trade-off between quality and frame rate creates a need for more advanced beamformers in plane wave imaging. Recently introduced convolutional or multiplication based beamforming techniques like the Filtered Delay Multiply And Sum (FDMAS) technique [5] yield interesting experimental results with improved lateral resolution and better contrast than Delay-And-Sum (DAS) beamforming.

COnvolutional Beamforming Algorithm (COBA) [6] brings a theoretical justification to these results, especially for sparse configurations, pointing out that they virtually create new elements on the probe array. Indeed, those methods multiply the signals received by the transducers elements pairwise, creating as many signals as the number of pairs of elements. Using a far field approximation, Regev et al. [6] showed that each obtained signal way is equivalent to the signal that would have been received by another (virtual) element.

Those methods work exclusively in the transducer elements dimension. We suggest a new compounding method, inspired by COBA but based on auto-convolutions in the angular dimension. This convolutional compounding method uses plane wave pressure field properties to virtually create new steering angles from a given set of plane waves.

Such a method aims at improving contrast and lateral resolution while being linear with respect to the tissue reflectivity function (TRF) and compatible with real-time implementations. It can be used with any given set of plane waves, complete or sparse.

In the following sections, we study theoretically the process of plane wave imaging and coherent compounding, and use this theoretical framework to analyse the proposed COnvolutional Compounding Algorithm (COCA) and its implications. We then test our method on the PICMUS dataset [7], with different angle sequences. Finally, we present and comment the obtained results in terms of contrast and resolution.

\section{Materials And Methods}

\section{A. Plane Wave Imaging and Coherent Compounding}

Plane wave imaging consists in transmitting unfocused plane waves by emitting almost simultaneously with all transducers. Such waves are then reflected by scatterers in the medium. Beamforming the backscattered echoes allows us to create a map of the tissue reflexivity function (TRF). With a single insonification, the whole medium is insonified and a low quality image can be obtained with a simple DAS algorithm.

In this work, we consider a point $P$ located at $\boldsymbol{r}=(x, z)$ in the medium. For the sake of simplicity, we suppose a uniform medium, with no absorption, a constant speed of sound $c$, and punctual transducers. Moreover, we derive the theoretical analysis for a unity amplitude, monochromatic wave of pulsation $\omega$.

The pressure field at point $P$ for a steered plane-wave insonification of angle $\alpha$ is of the form (as derived in [3]):

$$
p(t)=e^{j \omega t} e^{-j k(x \sin \alpha+z \cos \alpha)},
$$


where $k=\frac{\omega}{c}$ is the wave number.

This plane wave is reflected by point $P$ with TRF coefficient $\rho \in \mathbb{R}_{+}$. The reflected signal is received by all transducers, delayed, time gated, and summed according to the DAS algorithm to obtain the estimated TRF at $P: y$. The obtained value is proportional to the reflected pressure field.

If we emit a sequence of $N$ angles $\left(\alpha_{i}\right)_{0 \leq i \leq N-1}$, we obtain an array of estimated reflexivity $\boldsymbol{y}, y_{i}$ corresponding to the estimate of the TRF obtained at angle $\alpha_{i}, i=0, \ldots, N-1$. The coherent compounding method consists in delaying each plane wave to align phases on a focus point $F$ located at $\left(x_{F}, z_{F}\right)$. We obtain:

$$
y_{i}=C \rho e^{-j k\left(\left(x-x_{F}\right) \sin \alpha_{i}+\left(z-z_{F}\right) \cos \alpha_{i}\right)},
$$

with $C$ a complex constant depending on the reception focus pattern but not on $\alpha$.

Then, we take as final value $y_{c c}$, the average of all estimations:

$$
y_{c c}=\frac{1}{N} \sum_{i=0}^{N-1} y_{i}
$$

We will propose an alternative to coherent compounding that virtually creates new plane waves with different angles before summing them.

\section{B. Convolutional Compounding}

The idea of convolutional compounding is to increase the size of $\boldsymbol{y}$ by taking its autoconvolution. This technique is inspired by [6], but takes place along the angular dimension instead of the element one.

For linearity concerns, we begin by taking the square root of the modulus of our estimations, obtaining $s$ such that:

$$
s_{i}=\sqrt{\left|y_{i}\right|} e^{\phi\left(y_{i}\right)}, \quad i=0, \ldots, N-1,
$$

with $|$.$| the modulus and \phi($.$) the phase operator.$

We then take the autoconvolution of $s$ to obtain an augmented vector of estimations:

$$
\widetilde{y_{n}}=\boldsymbol{s} * \boldsymbol{s}[n]=\sum_{i+j=n} s_{i} s_{j}, \quad n=0, \ldots, 2 N-2 .
$$

Let us consider $n \in\{0, \ldots, 2 N-2\}$, and $i$ and $j$ natural numbers so that $i+j=n$. We want to study the expression of $s_{i} s_{j}$. After few calculations, we obtain:

$$
s_{i} s_{j}=\rho|C| e^{2 \phi(C)} \cdot e^{j 2 k \cos \left(\frac{\alpha_{i}-\alpha_{j}}{2}\right)\left(\left(x-x_{F}\right) \sin \widetilde{\alpha_{i, j}}+\left(z-z_{F}\right) \cos \widetilde{\alpha_{i, j}}\right),}
$$

with $\widetilde{\alpha_{i, j}}=\frac{\alpha_{i}+\alpha_{j}}{2}$.

For small angles, we can assume that $\cos \left(\frac{\alpha_{i}-\alpha_{j}}{2}\right) \approx 1$, such that (6) can be expressed as:

$$
s_{i} s_{j} \approx \rho|C| e^{2 \phi(C)} \cdot e^{j 2 k\left(\left(x-x_{F}\right) \sin \widetilde{\alpha_{i, j}}+\left(z-z_{F}\right) \cos \widetilde{\alpha_{i, j}}\right)},
$$

Using (2), we notice that (7) corresponds to the DAS estimate of the TRF for angle $\widetilde{\alpha_{i, j}}$ at frequency $2 \omega$, up to a constant phase shift.

Moreover, we observe that this new method is linear in the TRF as $\rho$ is left unchanged.

In a nutshell, the autoconvolution of an array of plane waves virtually creates new plane waves with half angles. The last step is then to coherently compound the estimates corresponding to the augmented set of plane waves, taking into account repetitions via weights for normalization purpose.

The final estimate is made of the sum of all virtually created angles. As the efficiency of coherent compounding lies in the number of angles summed, we want to calculate the number of obtained angles. From an set of $N$ angles, we create one new angle per pair of angles. The number of pairs in a set of cardinal $N$ is $\frac{N(N+1)}{2}$. However, as it is possible to obtain two times the same half angle from two different pairs, this number is only an upper bound to the actual number of obtained angles. Thus, for well chosen angles, we can reach 28 virtual angles from 7 or 45 from 9.

The whole COCA is described in Algorithm 1:



Output: Final estimation $y_{C O C A}$.

\section{Experimental Setup}

To validate the proposed method, we conduct several experiments. To do so, we use the IEEE IUS 2016 Planewave Imaging Challenge in Medical UltraSound (PICMUS) dataset presented in [7].

To assess our method, we use the two simulations, two in vitro and two in vivo acquisitions from PICMUS dataset.

The sequence is made 75 angles uniformly spread from $-16^{\circ}$ to $16^{\circ}$. Each wave is emitted with a 2.5 cycles transmit pulse of central frequency $5.208 \mathrm{MHz}$ and bandwidth $67 \%$.

For each experiment, we compare COCA and DAS with two different subsequences. The first one contains all 75 angles. The second one is made of 7 angles chosen among the 75 ones so that the cardinal of the augmented angle set (after autoconvolution) is maximal. Chosen and augmented angles are displayed Fig. 1.a and 1.b, respectively.

For each case, we beamform the signals with a F-number of $F_{\sharp}=1.75$, using a Tukey window with a $25 \%$ roll for apodization.

In simulations and in vitro experiments, we measure the axial and lateral resolution as well as the contrast. Resolution is assessed by the full width half maximum (FWHM). Contrast is measured with two metrics. Firstly, we use the contrast ratio 
a)



Fig. 1. Angles of emitted plane waves. a) Sequence of 7 angles emitted. b) Resulting sequence after autoconvolution. Original angles are filled.

$(C R)$, to obtain directly the difference between the gray level of the two regions, i.e. the difference of the estimated TRF in $\mathrm{dB}$. Secondly, we use the contrast to noise ratio (CNR), as defined in [7].

Each metric is assessed on several targets. For simulations, axial and lateral resolutions are measured on 20 scatterers and contrast on 9 anechoic objects. For in vitro experiments, resolutions are measured on 7 scatterers and contrast on 2 objects. We also measure the contrast ratio of the $3 \mathrm{~dB}$ and $-3 \mathrm{~dB}$ objects with respect to the background level in the second in vitro experiment to assess the linearity of COCA.

\section{RESUlts AND Discussion}

Beamformed images are displayed Fig. 2. We obtained 24 images, from 6 experiments, with two sequences (75 and 7 angles), with COCA and DAS. Measured lateral resolution and contrast ratio are reported in Table I.

We observe that COCA improves the lateral resolution compared to the DAS on both simulated and in vitro experiments. More precisely, we observe a 30\% improvement for the 75 angles sequence and a $15 \%$ for the 7 one. Obtained lateral resolution for the 7 angle sequence with COCA is only $3 \%$ worse than the lateral resolution obtained with the DAS algorithm and 75 angles..

Axial resolution is left unchanged by COCA. Indeed, this resolution only depends on physical phenomena as diffraction, or the length of the emitted pulse. Those phenomena happen before all non linear operations, for all angles, at pulsation $\omega$, and have no reason to be modified by COCA.

Regarding the contrast ratio, COCA gives an improvment of $6.6 \mathrm{~dB}$ in average for the 75 angles sequence and of $7.1 \mathrm{~dB}$ for the 7 angles one. The contrast ratio of COCA with the 7 angles sequence is not as good as the 75 angles DAS one, but it is at a difference of less than $4 \mathrm{~dB}$.

The CNR was also measured along with the CR. However, it is not significantly improved by COCA with respect to DAS. Indeed, COCA modifies the speckle, creating darker edges. Speckle signal does not follow a Rayleigh distribution

TABLE I

COMPARISON OF RESOLUTION AND CONTRAST.

\begin{tabular}{|c|c|c|c|c|c|c|c|}
\hline & \multicolumn{3}{|c|}{ Simulation } & \multicolumn{3}{|c|}{ In vitro } \\
\hline & & \multicolumn{2}{|c|}{$F W H M(\mathrm{~mm})$} & \multirow{2}{*}{$C R(d B)$} & \multicolumn{2}{|c|}{$F W H M(\mathrm{~mm})$} & \multirow{2}{*}{$C R(d B)$} \\
\hline & & Axial & Lateral & & Axial & Lateral & \\
\hline \multirow{2}{*}{$\begin{array}{c}75 \\
\text { angles }\end{array}$} & $\overline{\mathrm{DAS}}$ & 0.43 & 0.60 & 40.0 & 0.60 & 0.64 & 32.6 \\
\hline & COCA & 0.43 & 0.38 & 45.3 & 0.61 & 0.41 & 40.5 \\
\hline \multirow{2}{*}{$\begin{array}{c}7 \\
\text { angles }\end{array}$} & DAS & 0.43 & 0.72 & 28.2 & 0.60 & 0.78 & 23.7 \\
\hline & COCA & 0.43 & 0.61 & 36.2 & 0.60 & 0.66 & 29.9 \\
\hline
\end{tabular}

anymore, and the variance of speckle areas increases, leading to a lower CNR.

Nevertheless, the envelope mean value is unaltered, as shown by the measurement of $3 \mathrm{~dB}$ and $-3 \mathrm{~dB}$ objects contrast ratio which is almost constant. This contrast ratio does not depend on the emitted sequence nor on the algorithm used: CR differs by less than $0.3 \mathrm{~dB}$ in all four pictures. This shows that COCA is indeed linear in the TRF.

Finally, in vivo images validate the effect of COCA. The edges look more defined due to the non linearity and a better focusing in transmit induced by the higher number of waves. Compared to DAS pictures, the contrast looks higher, and clutter is reduced by the increase in the number of angles. By visual assessment, we notice that the last rightmost image corresponding to COCA with 7 angles is of similar quality to the leftmost one corresponding to the DAS algorithm with 75 angles.

\section{CONCLUSION}

In this paper, we present a new compounding technique based on auto-convolution. We see that this technique virtually creates new angles from a set of steered plane waves, and thus can be used to reduce the number of emissions and increase the frame rate.

Experiments on PICMUS dataset show that this technique improves contrast and resolution, while maintaining linearity properties with respect to the TRF. Results point out that this algorithm applied to a well chosen 7 angles sequence can yield very good results, with a resolution as good as the 75 angles DAS reference, and contrast less than $4 \mathrm{~dB}$ lower.

However, as for most of non-linear technique, this method alters the speckle pattern.

For future work, effect of angular apodization and methods for sparse sequences generation will be studied and performance will be evaluated on other geometries as well, e.g. convex or phased arrays.

\section{REFERENCES}

[1] J. Bercoff, "Ultrafast Ultrasound Imaging," in Ultrasound Imaging - Med. Appl. InTech, aug 2011.

[2] M. Tanter and M. Fink, "Ultrafast Imaging in Biomedical Ultrasound," IEEE Trans. Ultrason. Ferroelectr. Freq. Control, vol. 61, no. 1, pp. 102119 , jan 2014.

[3] G. Montaldo, M. Tanter, J. Bercoff, N. Benech, and M. Fink, "Coherent Plane-Wave Compounding for Very High Frame Rate Ultrasonography and Transient Elastography," IEEE Trans. Ultrason. Ferroelectr. Freq. Control, vol. 56, no. 3, pp. 489-506, mar 2009.

[4] B. Denarie, T. A. Tangen, I. K. Ekroll, N. Rolim, H. Torp, T. Bjastad, and L. Lovstakken, "Coherent Plane Wave Compounding for Very High Frame Rate Ultrasonography of Rapidly Moving Targets," IEEE Trans. Med. Imaging, vol. 32, no. 7, pp. 1265-1276, jul 2013.

[5] G. Matrone, A. S. Savoia, G. Caliano, and G. Magenes, "The Delay Multiply and Sum Beamforming Algorithm in Ultrasound B-Mode Medical Imaging," IEEE Trans. Med. Imaging, vol. 34, no. 4, pp. 940-949, apr 2015.

[6] R. Cohen and Y. C. Eldar, "Sparse Convolutional Beamforming for Ultrasound Imaging," IEEE Trans. Ultrason. Ferroelectr. Freq. Control, vol. 65, no. 12, pp. 2390-2406, dec 2018.

[7] H. Liebgott, A. Rodriguez-Molares, F. Cervenansky, J. Jensen, and O. Bernard, "Plane-Wave Imaging Challenge in Medical Ultrasound," in 2016 IEEE Int. Ultrason. Symp. IEEE, sep 2016. 
a) DAS, 75 PWs
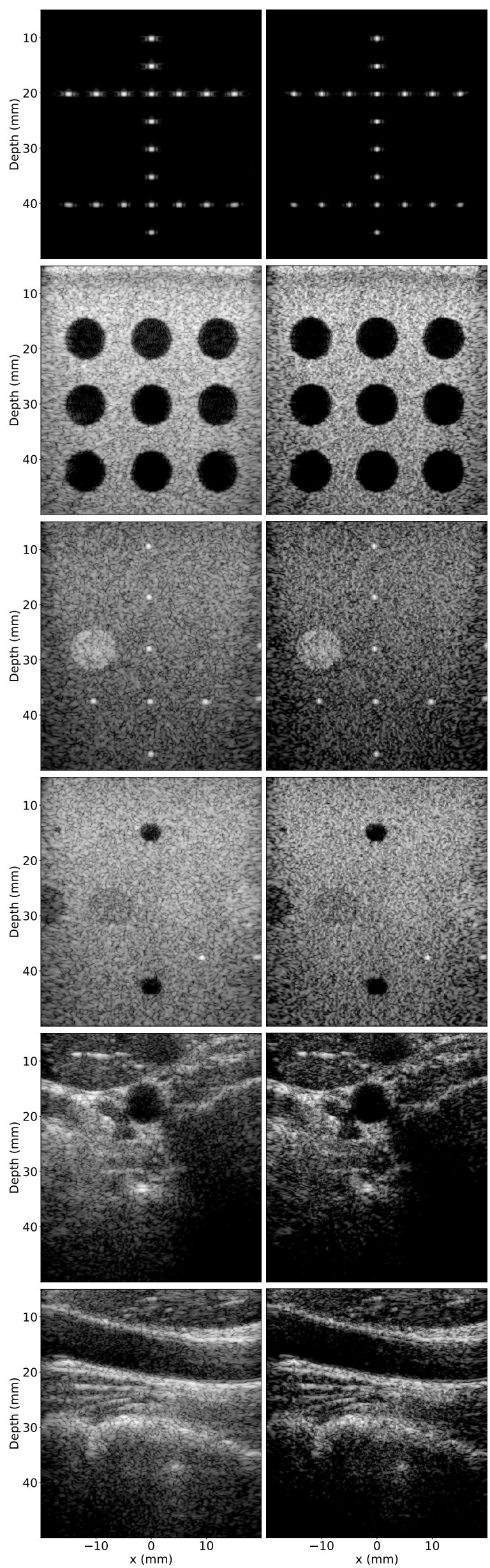

c) DAS, 7 PWs
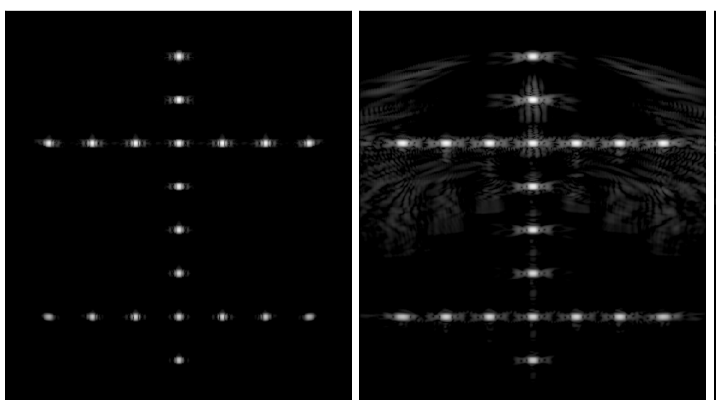

d) COCA, 7 PWs
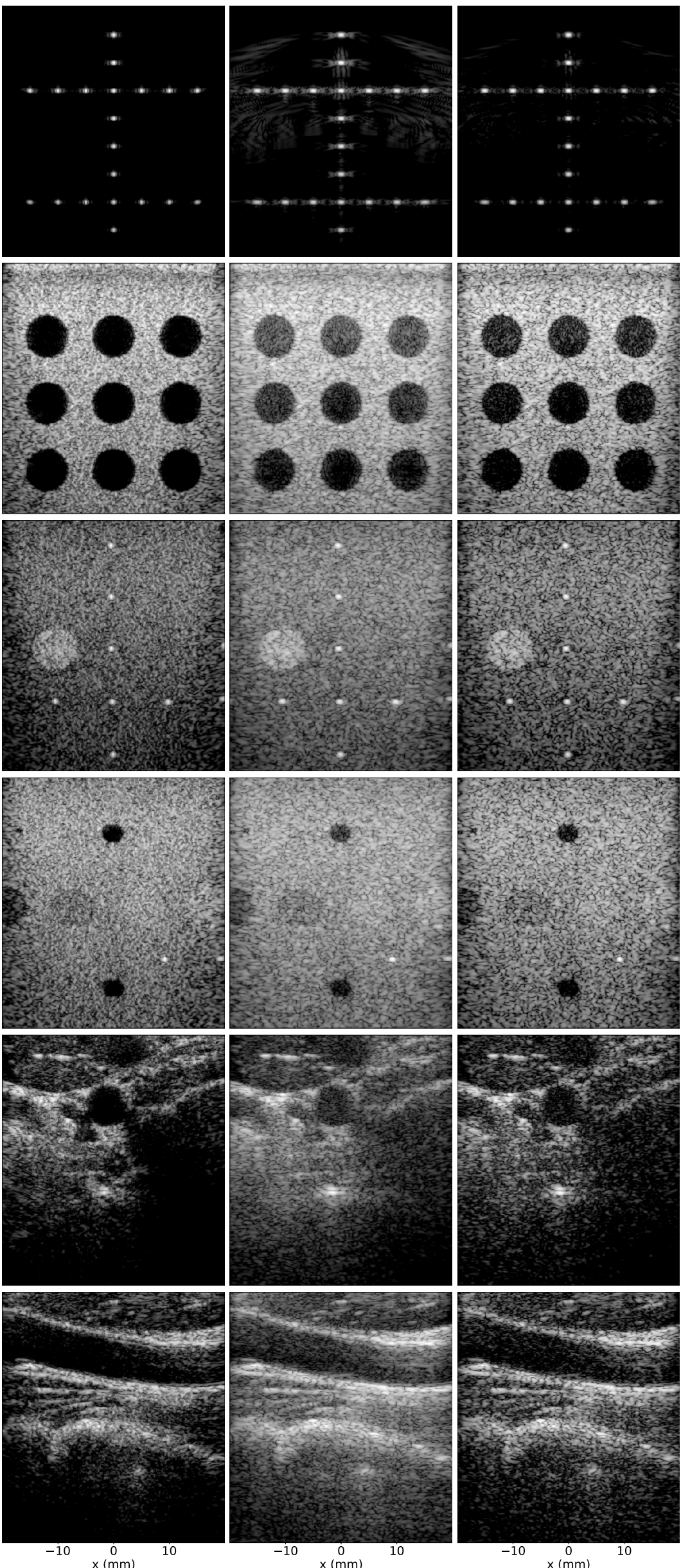

Fig. 2. Obtained images of simulated, in vitro and in vivo experiments. By column, from left to right: a) DAS with 75 angles, b) COCA with 75 angles, c) DAS with 7 angles, d) COCA with 7 angles. All pictures are displayed with a $60 \mathrm{~dB}$ dynamic range. 